\title{
Adoption of technology-enabled care for patients with respiratory conditions in primary care
}

\author{
Ruth Chambers, Marc Talbot, Rachel Hatfield
}

\section{Citation}

Chambers $\mathrm{R}$, Talbot M, Hatfield $\mathrm{R}$ (2019) Adoption of technologyenabled care for patients with respiratory conditions in primary care. Primary Health Care. doi: 10.7748/phc.2019.e1551

\section{Peer review}

This article has been subject to external double-blind peer review and has been checked for plagiarism using automated software

\section{Correspondence}

ruth.chambers@stoke.nhs.uk

\section{Conflict of interest}

None declared

\section{Accepted}

13 March 2019

\section{Published online} June 2019

\section{Gopen access}

This is an open access article distributed under the terms of the Creative Commons Attribution-Non Commercial 4.0 International (CC BY-NC 4.0) licence (see https:// creativecommons.org/licenses/ by-nc/4.0/), which permits others to copy and redistribute in any medium or format, remix, transform and build on this work non-commercially, provided appropriate credit is given and any changes made indicated

\begin{abstract}
A county-wide programme to promote interest in and adoption of technology-enabled care (TEC) by general practice teams focused on take-up for common health conditions where there is evidence of unwarranted clinical variation, such as asthma and chronic obstructive pulmonary disease. Comments and perspectives were gathered from hundreds of delegates at workshops that combined upskilling in clinical management and a range of methods of digitally providing care, and from general practice nurses who participated in digital upskilling action learning sets.

Clinicians strongly committed to providing care digitally in frontline general practice. The benefits envisaged included greater productivity, improved clinical outcomes and empowering patients to care for themselves more. TEC can help to minimise unwarranted clinical variation and improve care for respiratory conditions across patient populations, for example.
\end{abstract}

\section{Author details}

Ruth Chambers, professor of healthcare, Keele University, NHS Stoke on Trent Clinical Commissioning Group, Hanley, England; Marc Talbot, programme manager, NHS Midlands and Lancashire Commissioning Support Unit, Lancashire, England; Rachel Hatfield, national project manager, General Practice Nursing Action Learning Sets, Hanley, England

\section{Keywords}

asthma, chronic obstructive pulmonary disease, communication, health literacy, information technology, patient empowerment, personalisation, service improvement, telehealth

\section{Introduction}

Recent national analysis (Salciccioli et al 2018) highlights that life expectancy and mortality rates are no longer improving year on year. They may even be worsening for some people, such as younger people and those with chronic respiratory diseases. Other downward trends reflect the effects of winter and periods of cold weather on mortality rates (Public Health England (PHE) 2018).

Chronic obstructive pulmonary disease (COPD) is now the fourth greatest cause of death for people of all ages in the UK (Davies 2018). Changing unhealthy lifestyle behaviours, such as smoking, could prevent up to $80 \%$ of new cases of heart disease, stroke, type 2 diabetes and many cancers (Davies 2018). Multimorbidity is increasingly becoming a problem for the NHS, particularly general practice, as more patients are aged over 65 . There is an urgent need to deliver care more productively and efficiently to accommodate this escalating demand. The overall aim for sustainability and transformation partnerships (STPs) and integrated care systems (ICSs) must be to redress unwarranted clinical variations in the delivery of care.

NHS England's (2019) Long Term Plan sets out its ambitions for the health and care service, and promises additional funding for the next ten years to enable people and practices to benefit from the latest digital technologies. The plan states that all patients will have access to their care plans and communications from their care professionals through an NHS app by 2021. In addition, digitally-enabled primary and outpatient care, in which patients will be cared for and supported at home using remote monitoring and digital tools, will go mainstream across the NHS by 2024 .

The King's Fund (2019) highlights the need for digital infrastructure, and for patients and the workforce to be supported with digital tools, because these capabilities enable teams to care for patients better and continuously improve the population's health. 
A partnership between professionals and the public is needed to encourage patients to manage their own health, which is part of a broader cultural change resulting in a 'shared responsibility for health'.

The Topol Review (Health Education England 2019) includes recommendations about the workforce element of the NHS Long Term Plan. It notes that the workforce and the population are changing, and have new expectations, and that digital healthcare technology should address the healthcare challenges of the 21 st century. It also suggests that new technologies will not replace healthcare professionals, but will enhance their care, giving them more time to look after patients, improve outcomes and empower patients to participate more in their own care.

The adoption of innovative digital technologies in health and social care pathways is often poorly coordinated and inconsistent (Ramanauskas 2019). In England, new schemes are often run as time-limited pilots, with insufficient budgets and shortterm planning, and so do not enable systemwide change or widespread adoption. Many clinicians view patient-centred technology with suspicion. For example, an early trial of expensive equipment in patients' homes was found to require broadband and a 24/7 monitoring service. When all additional charges were considered, the cost-benefits became unclear (Henderson et al 2013).

\section{Background}

The programme described in this article involved readily available, free or low-cost technology that can empower patients to take more responsibility for their health and wellbeing without relying on a $24 / 7$ monitoring service. Technology-enabled care (TEC) will be vital to the sustainability of the NHS and social care if and when it is adopted as usual practice at scale. TEC should help to alleviate demands on the NHS by providing delivery methods that help practitioners to substitute remote care for face-to-face consultations when they are justified. This includes speeding up follow-on care, such as sharing and acting on test results, and encouraging people to take more responsibility for their health conditions and lifestyle habits, thereby reducing pressure on frontline health services.

Over the past two years, Staffordshire STP's digital workstream has been introducing a TEC programme aligned to the national ambitions for STPs (King's Fund 2017) for a patient population of 1.2 million registered at 155 general practices.
The programme focuses on strengthening prevention and early intervention, improving productivity, tackling variations in care, developing enablers, upskilling the workforce, and redesigning and improving healthcare services in all settings. It promotes healthy living and lifestyle choices through trusted mobile apps, and telehealth interactions between patients and clinicians. It also uses social media to engage with patients across the county and in peer support groups, and encourage them to care for themselves.

The aim of this TEC county-wide programme was to digitally enable general practice at scale. It focuses on clinical variations, such as those in care for respiratory conditions and associated clinical outcomes.

TEC could reduce clinical variations in care and alleviate demands on services by providing healthcare professionals with easily accessible tools for treating patients and improve productivity. But if TEC is implemented without enough investment in equipment, or in enabling the digital inclusion of patients, carers, clinicians and managers, it could exacerbate health inequalities, variations in care and associated outcomes (National Institute for Health and Care Excellence (NICE) 2018).

\section{Methods}

Technology for the programme, such as video consultation, trusted health and wellbeing-related apps, social media and webbased resources supporting care of long-term conditions (LTCs) and redressing adverse lifestyle habits, was picked for affordability or availability. Some popular, relatively low-cost or nationally-funded commercial applications, such as Flo simple telehealth interactive texting, AliveCor's KardiaMobile device for screening for atrial fibrillation and online clinical consultation tools, were also included.

\section{Digital upskilling of clinicians}

A pilot to digitally upskill general practice nurses (GPNs) to create digital champions was undertaken across Staffordshire between March and November 2018. Forty local GPNs participated in six parallel action learning sets (ALS) to learn to adopt and embed TEC to provide substantive cost, clinical and quality benefits in their practices and subsequently the local health economy. The GPNs each attended three ALS sessions to learn how to use and implement different modes of digital technology to promote patients' health and well-being and prevent deterioration of their health conditions. 
The ALS programme is funded by NHS England and supports its aims for general practice (NHS England 2016, 2017) and PHE's (2019) framework. The ALS pilot has been extended to upskill 120 GPNs in 12 cohorts across England.

Learning has been shared at a specially created national conference and through published papers. These include case studies that demonstrate how clinicians using TEC can support patients to understand their conditions, adopt and access preventative interventions, and increase access to selfcare information and shared management of long-term care (Chambers et al 2018a, 2018b, 2018c, Johnson et al 2018).

\section{Digital champions and exemplar} general practices

This programme sought to establish:

» Thirty 'digital champion' practices providing at least two TEC services to patients for at least three months.

» Ten 'digital exemplar' practices providing at least three TEC services to patients for at least six months.

A digital expert social enterprise trained individual clinicians and practice teams, and provided essential equipment.

Clinical leadership and facilitation was provided by the STP.

\section{Underpinning care pathways}

Learning resources included three workshops run across Staffordshire for general practice clinicians and managers that focused on the clinical management of COPD and asthma, alongside relevant TEC. There were also three parallel workshops on clinical upskilling in the management of AF and hypertension, and relevant TEC. These demonstrated the potential benefits to patients and healthcare professionals through case studies illustrating practice productivity, adherence to medication or other interventions and the convenience of remote delivery of general practice care.

Feedback from delegates at workshops and participants in the GPN digital upskilling programme enabled learning to be captured. This feedback informed pilots using TEC to enable long-term care pathways spanning general practices, and all health and care settings.

Reports were produced in October 2018 that highlighted each of the 155 practices' clinical variation against local and national clinical indicators. Subsequent workshops, between November 2018 and January 2019, demonstrated how specific modes of easily accessible TEC can help to reduce unwarranted variation. It was also shown how TEC implemented for specific cohorts of patients can help them manage their LTCs and adverse lifestyle habits, and can support practice teams in improving below average quality indicators.

The workshops also showed how specific types of TEC could help patients to make informed decisions about how to manage their own health conditions.

\section{Digital inclusion of patients}

Digital inclusion of patients and members of the public was promoted in a digital inclusion national pathfinder that piloted upskilling in dementia cafés and other community centres. This was awarded by the Good Things Foundation (NHS Stoke-on-Trent Clinical Commissioning Group 2018).

\section{Results}

About 300 attendees at the workshops and national conference gave their personal perspectives of using TEC in their practices or other settings, and of how digital enablement has helped them to interact with patients and carers and improved the care they provide. Boxes 1 and 2 show the main challenges and opportunities they reported.

\section{Box I. Challenges}

Clinicians' concerns about technology-enabled care (TEC) can be grouped into four themes: Integrated working

॥ There is a lack of integration between TEC services and local and national healthcare services

॥ TEC will not generate its expected benefits if patients and practices are not linked to healthcare services in the wider health economy, for example through integrated care records, or collaboration between GPs and community or acute healthcare settings

\section{Inequalities}

॥ TEC opportunities provided by sustainability and transformation partnerships (STPS) and integrated care systems (ICSs) can be inconsistent, which can result in different TEC upskilling and implementation practices

》 There is inconsistency in TEC funding across and between STPS and ICSs

\section{Upskilling of clinicians and succession planning}

॥ There is a need for funding specifically for digital technology training and implementation

॥ There is a need for succession planning in practice teams, and across STPS and ICSs' programme leads and managers, to ensure TEC experiences and knowledge are retained and cascaded

॥ Clinicians are often nervous when commencing implementation of TEC, but were surprised by how easy using TEC is once they started

\section{Engagement}

॥ The availability locally of TEC to clinicians, managers, patients and carers is not being promoted enough

》 Approaches to engage individual patients in TEC can be time-consuming

॥ Some patients are keener than others to use TEC in their healthcare

॥ There is a need to identify the forms of TEC most suitable for specific patients or cohorts, and tailor care to the individual or according to its purpose

I Some patients and carers cannot access, and not all patients are ready to embrace, all forms of TEC

》) If specific types of TEC are discontinued, there is a risk of creating barriers to patient use 
A mix of GPNs, GPs and managers who participated in workshops gave feedback on the potential use of relevant TEC with patients with asthma or COPD (Box 3). Many practice teams subsequently requested TEC training and development.

Those attending the workshops discussed variations in the populations of the six Staffordshire clinical commissioning groups

\section{Box 2. Opportunities}

The opportunities offered by technology-enabled care (TEC) can be grouped into four themes:

Patient engagement

॥ Using TEC to communicate information enables clinicians to engage with patients who have been unable or unwilling to attend face-to-face appointments

I) The number of missed appointments has reduced because TEC offers an alternative to face-to-face appointments

I Patients are often willing to involve their wider support network, including their family and carers, when using TEC

\section{Patient empowerment}

॥ TEC improves patients' self-awareness and increases their control of their conditions, making them more willing to take responsibility for their long-term care and adverse lifestyle habits

॥ Patients can take a more central role in the management of their conditions through self-education and can develop greater knowledge of their conditions through the use of trusted apps. This encourages a sense of ownership of their healthcare

I) Many patients use similar technologies in their daily lives and so are receptive to using TEC

Enhanced productivity

॥ The number of missed appointments with clinicians has been reduced

॥ Patients using TEC are willing to communicate with clinicians only where appropriate, which avoids unnecessary face-to-face consultations

II Interactive texting and video consultations are especially useful in reducing the number of missed appointments, as well as in engaging previously unreceptive patients

\section{Improving clinical outcomes}

I) When agreed treatment protocols are in place, clinical safety is enhanced, which makes patients and the clinical team feel comfortable about shared management

॥ Wider practice support is necessary to continue the adoption of TEC across general practices, and to ensure succession planning

» When TEC underpins agreed shared management of health conditions, patients and clinicians feel they are working together. This enhances adherence to medication, for example, which in turn improves clinical outcomes

\section{Box 3. Feedback on using technology-enabled care}

Responses to the question 'How useful has technology-enabled care (TEC) been or might be in improving the care of patients with respiratory conditions in your practice?'

॥ 'TEC gives me the ability to give patients information and reinforcement on how to manage their asthma following asthma reviews, and to remind them of inhaler technique' (practice nurse 1)

II 'I would like to persuade the practice nurse team to use Flo Simple Telehealth to engage younger asthmatic patients' (GP 1)

I) 'The Manage Your Health app is fantastic to help educate and give patients information' (practice nurse 2)

॥ 'TEC empowers patients. It lets them know more about their condition and manage it in simple ways, which makes them proud' (practice nurse 3 )

/) 'Flo telehealth is a good way to monitor patients' blood pressure and chronic obstructive pulmonary disease (COPD)' (trust quality improvement nurse manager)

॥ 'I want to use Flo telehealth for our patients with COPD. We currently have no modes of TEC services in our surgery though' (GP 2)

I) 'We use TEC services to empower patients to self-manage. We contact them with daily reminders about, and support and advice for, their chronic illnesses' (practice manager) and how practices should address low achievement through comparisons with national averages (Table 1 ).

Each practice received a tailored report comparing its averages to those of the CCG and nationally. The report then identified possible types of TEC the practice could implement to improve clinical asthma outcomes and reduce the annual cost of hospital admissions for patients with asthma (Box 4).

Fourteen of the 29 GPNs who took part in the ALSs chose to use TEC mainly with patients who had asthma and/or COPD, rather than other long-term conditions. The modes of TEC they were using six months later are shown in Table 2.

\section{Discussion}

Three years after the inception of STPs, the landscape continues to evolve, with the emergence of new models of care, including ICSs and accountable care systems (ACSs), that aim to bring about greater integration of health and social care services. There is a lack of integration between health and care services, however, with NHS organisations tending to work on digital enablement in silos. This lack of integration often leads to patients being unaware of TEC and services, and fewer opportunities for collaborative working between care providers.

The TEC programme in Staffordshire has started to bridge this gap by increasing practice teams' engagement with, and deployment of, TEC, and their interaction with other organisations in the local healthcare economy in working towards a digitally transformed NHS. The programme has helped to increase patient engagement through the use of technology, allowing them to access healthcare services in several new ways.

As a result, the number of patients taking a more active role in their healthcare treatment and the management of their LTCs is increasing. TEC is also addressing operational issues in primary care by enhancing clinical productivity, leading to fewer missed appointments and more efficient communication with patients. The programme has also increased engagement between patients and clinicians, which has led to improved clinical outcomes.

As Table 1 indicates, there is much work to do nationally and across the STP to reduce health inequalities in LTCs, such as COPD (NICE 2018). Practice reports highlight the disparities in this area, but also 
suggest how they can be redressed through digital delivery.

Table 2 shows the effectiveness of digital upskilling clinicians and how their appetite for TEC increases as they realise its benefits. This results in greater adoption of TEC, leading to a more robust, transformational approach to tackling the prevalence and treatment of, for example, respiratory condition.

The national analysis of trends in mortality rates has highlighted the importance of increased efforts to reduce patients' risks from the main LTCs by addressing the underlying determinants of health and reducing adverse lifestyle factors, such as smoking and obesity (Gabel et al 2018, PHE 2018). The analysis emphasises the need to proactively support the most vulnerable in society, particularly older people, to minimise the effects of preventable deterioration in their health.

The population health management approach described in this article, particularly in relation to the capture of comparative data and its use in improving services, should underpin the intelligent commissioning of healthcare services, such as pulmonary rehabilitation services, as more people are diagnosed and managed with COPD. Once patients are using TECS such as Flo Simple Telehealth, they are empowered to share the management of their LTCs with their clinicians.

Table I. Staffordshire Clinical Commissioning Group comparative data for respiratory conditions and smoking 2017-18

\begin{tabular}{|l|c|c|c|}
\hline $\begin{array}{l}\text { Data } \\
\text { source }\end{array}$ & $\begin{array}{c}\text { Percentage of adults } \\
\text { With chronic obstructive } \\
\text { pulmonary disease who were } \\
\text { immunised for influenza between } \\
\text { 1 August 2017 and 31 March 2018 }\end{array}$ & $\begin{array}{c}\text { With asthma who had received reviews } \\
\text { that included the three Royal College of } \\
\text { Physicians questions to assess asthma } \\
\text { control over the previous year }\end{array}$ & $\begin{array}{c}\text { Who had been offered } \\
\text { smoking cessation } \\
\text { support over the } \\
\text { previous year }\end{array}$ \\
\hline CCG1 & 98.0 & 75.6 & 86.4 \\
\hline CCG 2 & 97.8 & 76.6 & 87.2 \\
\hline CCG 3 & 98.6 & 77.6 & 91.7 \\
\hline CCG 4 & 97.5 & 75.3 & 90.6 \\
\hline CCG 5 & 96.8 & 77.8 & 86.7 \\
\hline CCG 6 & 98.7 & 75.6 & 87.1 \\
\hline $\begin{array}{l}\text { National } \\
\text { average }\end{array}$ & 97.4 & 75.9 & 89.2 \\
\hline (Pearson \& Bucknall 1999, NHS Digital 2018) & & \\
\hline
\end{tabular}

\section{Box 4. Example narrative for asthma from an individualised practice report}

\section{Asthma prevalence}

'Low $(<5.7 \%)$. The number of patients diagnosed with, and coded as having, asthma is low compared with your clinical commissioning group's (CCG) average prevalence rate. The CCG average is similar to the national average. You could consider using technology-enabled care (TEC) to increase patients' awareness, for example through your practice website or with public Facebook messaging'

\section{Asthma non-elective admission spend 2016-17}

'The spend calculated for in-hospital asthma care of adult patients registered with your practice provides an idea of potential savings if the deterioration of their asthma can be prevented. You could consider using TEC to enhance their ongoing clinical care by recommending apps, such as Manage Your Health, with which they can watch avatars demonstrating how to use inhalers properly; Flo telehealth interactive texting, which can help patients take their medications and improves clinical management; or video consultations with patients who find attending asthma reviews difficult'

\section{Asthma review in the past 12 months}

'Low $(<75 \%)$. You could consider using TEC, such as your practice website, public Facebook messaging and Flo telehealth interactive texting, to help patients understand the importance of their asthma reviews, and of other health conditions and lifestyle habits' 
One example of this was a Flo trial in a large inner-city practice in which 25 patients with COPD were signed up to Flo, after which only two were admitted to hospital during the following winter (Collis et al 2014).

The deployment of TEC in the local health economy enhances the quality and diversity of healthcare services offered to patients. Meanwhile, clinicians have developed an appetite for continued implementation and upscaling of TEC that has been endorsed by their fellow healthcare professionals.

Table 2. Technology-enabled care used with patients with asthma and/or chronic obstructive pulmonary disease

\begin{tabular}{|l|l|l|}
\hline Method & Asthma & COPD \\
\hline Closed Facebook group & 5 & 0 \\
\hline Trusted app & 9 & 7 \\
\hline Flo simple telehealth & 4 & 3 \\
\hline Video consultation & 1 & 1 \\
\hline
\end{tabular}

\section{Conclusion}

Staffordshire STP's TEC programme has demonstrated the need for commitment to digital solutions that enable systemwide transformation of healthcare. The benefits of giving clinicians and patients access to TEC for the main LTCs and the necessary digital upskilling include enhanced productivity, improved clinical outcomes and patient empowerment.

TEC increases engagement between clinicians and patients by offering alternatives to face-to-face appointments, saving patients and clinicians time, and enabling clinicians to engage with patients previously unreceptive to best practice.

Patients with more opportunities to self-manage their health conditions become empowered and take a more central role in their healthcare, generating improved clinical outcomes. In addition, TEC can minimise unwarranted clinical variation and improve clinical outcomes across STP-based patient populations, through the willingness of front-line staff who recognise the challenges and the need to adopt TEC at scale.

\section{References}

Chambers R, Cox T, Hughes A et al (2018a) Technology enabled care services for patients with long-term conditions. Journal of General Practice Nursing. 4, 3, 60-64

Chambers R, Hughes A, Beaney P et al (2018b) You too can be a digital practice nurse champion. Practice Nurse. 48, 6, 11-15.

Chambers R, McKinney R, Schmid M et al (2018c) Digital by choice: becoming part of a digitally ready general practice team. Primary Health Care. $28,7,22-27$.

Collis L, Whitmore M, Chambers R (2014) Telehealth in primary care: what's in it for me? Practice Nursing. 25, 8, 406-408.

Davies S (2018) Annual Report of the Chief Medical Officer, 2018: Health 2040 - Better Health within Reach. Department of Health and Social Care, London.

Gabel F, Chambers R, Cox T et al (2018) An evaluation of a multifaceted, local Quality Improvement
Framework for long-term conditions in UK primary care. Family Practice. doi: 10.1093/fampra/cmy128

Health Education England (2019) The Topol Review: Preparing the Healthcare Workforce to Deliver the Digital Future. topol.hee.nhs.uk/ wp-content/uploads/HEE-Topol-Review-2019.pdf (Last accessed: 31 May 2019.)

Henderson C, Knapp M, Fernández J-L et al (2013) Cost effectiveness of telehealth for patients with long term conditions (Whole Systems Demonstrator telehealth questionnaire study): nested economic evaluation in a pragmatic, cluster randomised controlled trial. BMJ. doi: org/10.1136/bmi.f1035

Johnson K, Johnson H, McGougan T (2018) The role of technology-enabled care in high-quality patient care. Practice Nursing. 29, 8, 397-399

King's Fund (2017) Sustainability and Transformation Plans (STPs) Explained. King's Fund, London.

King's Fund (2019) The NHS Long-Term Plan Explained. King's Fund, London.
NHS Digital (2018) Quality and Outcomes Framework, Achievement, Prevalence and Exceptions Data - 2017-18 [PAS]. digital.nhs.uk/ data-and-information/publications/statistica/ quality-and-outcomes-framework-achievementprevalence-and-exceptions-data/2017-18 (Last accessed: 31 May 2019)

NHS England (2016) General Practice Forward View. NHS England, London

NHS England (2017) General Practice - Developing Confidence, Capability and Capacity: A Ten Point Action Plan for General Practice Nursing. NHS England, London.

NHS England (2019) A Five-Year Framework for GP Contract Reform to Implement the NHS Long Term Plan. NHS England, London.

NHS Stoke-on-Trent CCG (2018) Spotlight on Digital Health: Long Term Conditions. www.clinitecs.uk/ spotlight-on-digital-health-long-term-conditions (Last accessed: 22 May 2019.)
National Institute for Health and Care Excellence (2018) Chronic Obstructive Pulmonary Disease in Over 16s: Diagnosis and Management. NICE, London.

Pearson M, Bucknall C (1999) Measuring Clinical Outcome in Asthma: a Patient-focused Approach. Clinical Effectiveness \& Evaluation Unit. Royal College of Physicians, London.

Public Health England (2018) A Review of Recent Trends in Mortality in England. PHE, London.

Public Health England (2019) All Our Health: About the Framework. PHE, London.

Ramanauskas B (2019) Embracing Technology in Health and Social Care. Tax Payers' Alliance, London.

Salciccioli J, Marshall D, Shalhoub J et al (2018) Respiratory disease mortality in the United Kingdom compared with EU15+ countries in 1985-2015: observational study. BMJ. doi: org/10.1136/bmj.k4680 